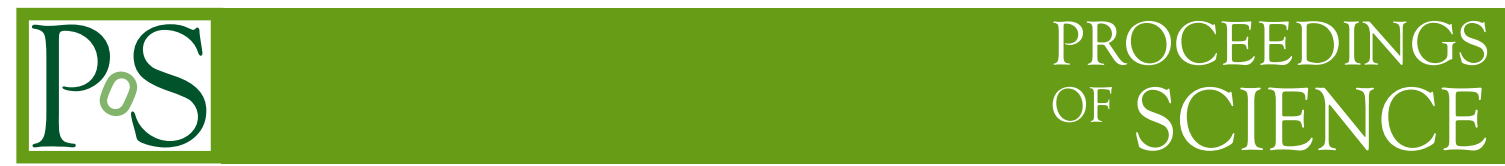

\title{
tops at NLO and NNLO
}

\section{Michal Czakon*t}

Institut für Theoretische Physik E, RWTH Aachen University D-52056 Aachen, Germany

E-mail: mczakon@physik.rwth-aachen.de

We briefly review the recent progress in the study of higher order corrections to hadron scattering processes involving top quark pairs in the final state. In particular, we discuss the Monte-Carlo simulation of the $p p \rightarrow t \bar{t} b \bar{b}$ process at the next-to-leading order with the help of the Helac-NLO system, and the status of the soft-gluon resummation program for $p p \rightarrow t \bar{t}$ near threshold at the next-to-next-to-leading order.

RADCOR 2009 - 9th International Symposium on Radiative Corrections (Applications of Quantum Field Theory to Phenomenology)

October 25-30 2009

Ascona, Switzerland

\footnotetext{
${ }^{*}$ Speaker.

${ }^{\dagger}$ This work was supported by the Heisenberg Programme of the Deutsche Forschungsgemeinschaft.
} 


\section{Introduction}

Production of top quark pairs in hadronic collision, i.e. at the TeVatron and LHC, is and will certainly remain an attractive subject of study. From the multitude of problems, two correspond to the current forefront of science: top pair production with two additional partons at the next-toleading order of QCD, and top pair production with up to two partons at the next-to-next-to-leading order. Both problems have generated interesting advances in theory. Below, we shall summarize the current status.

\section{NLO}

In this proceedings contribution, we present the results obtained with the help of the Helac-NLO system. This software consists of the following: Helac-Phegas [1], Helac-1Loop [2], based on the OPP method and CutTools [3], and finally Helac-Dipoles [4].

We consider the process $p p \rightarrow t \bar{t} b \bar{b}+X$ at NLO [5, 6] at the LHC, i.e. for $\sqrt{s}=14 \mathrm{TeV}$. For the top-quark mass, renormalized in the on-shell scheme, we take the numerical value $m_{t}=172.6 \mathrm{GeV}$. All other QCD partons including $b$ quarks are treated as massless particles. All final-state $b$ quarks and gluons with pseudorapidity $|\eta|<5$ are recombined into jets with separation $\sqrt{\Delta \phi^{2}+\Delta y^{2}}>$ $D=0.8$ in the rapidity-azimuthal-angle plane via the IR-safe $k_{T}$-algorithm. Moreover, we impose the following additional cuts on the transverse momenta and the rapidity of two recombined b-jets: $p_{T, b}>20 \mathrm{GeV},\left|y_{b}\right|<2.5$. The outgoing (anti)top quarks are neither affected by the jet algorithm nor by phase-space cuts.

We consistently use the CTEQ6 set of parton distribution functions (PDFs), i.e. we take CTEQ6L1 PDFs with a 1-loop running $\alpha_{s}$ in LO and CTEQ6M PDFs with a 2-loop running $\alpha_{s}$ in NLO, but the suppressed contribution from $b$ quarks in the initial state has been neglected. The number of active flavors is $N_{F}=5$, and the respective QCD parameters are $\Lambda_{5}^{L O}=165 \mathrm{MeV}$ and $\Lambda_{5}^{M S}=226 \mathrm{MeV}$. In the renormalization of the strong coupling constant, the top-quark loop in the gluon self-energy is subtracted at zero momentum. In this scheme the running of $\alpha_{s}$ is generated solely by the contributions of the light-quark and gluon loops. By default, we set the renormalization and factorization scales, $\mu_{R}$ and $\mu_{F}$, to the common value $\mu_{0}=m_{t}$.

With these parameters and assumptions we [6] obtain the following results for the cross sections at LO and NLO, which are in excellent agreement with [5]

$$
\begin{gathered}
\sigma_{t \bar{t} b \bar{b}}^{\mathrm{LO}}\left(\mathrm{LHC}, m_{t}=176.2 \mathrm{GeV}, \mathrm{CTEQ6L1}\right)=1489.2_{-565.8(38 \%)}^{+1036.8(70 \%)} \mathrm{fb}, \\
\sigma_{t \bar{t} b \bar{b}}^{\mathrm{NLO}}\left(\mathrm{LHC}, m_{t}=176.2 \mathrm{GeV}, \mathrm{CTEQ} 6 \mathrm{M}\right)=2636_{-703(27 \%)}^{+862(33 \%)} \mathrm{fb} .
\end{gathered}
$$

Besides the agreement for the cross sections, we were also interested in distributions. As far as internal consistency checks are concerned, we used the independence of the results on the size of the subtraction phase space in the dipole formalism. This led to the plots presented in Fig. 1. The notation and the definition of the phase space cut-off parameter $\alpha_{\max }$ is given in the original publication.

The most important result of the study are the distributions presented in Fig. 2. They show the size of the differential $\mathrm{K}$ factors, as well as the shape changes in the distributions themselves. 

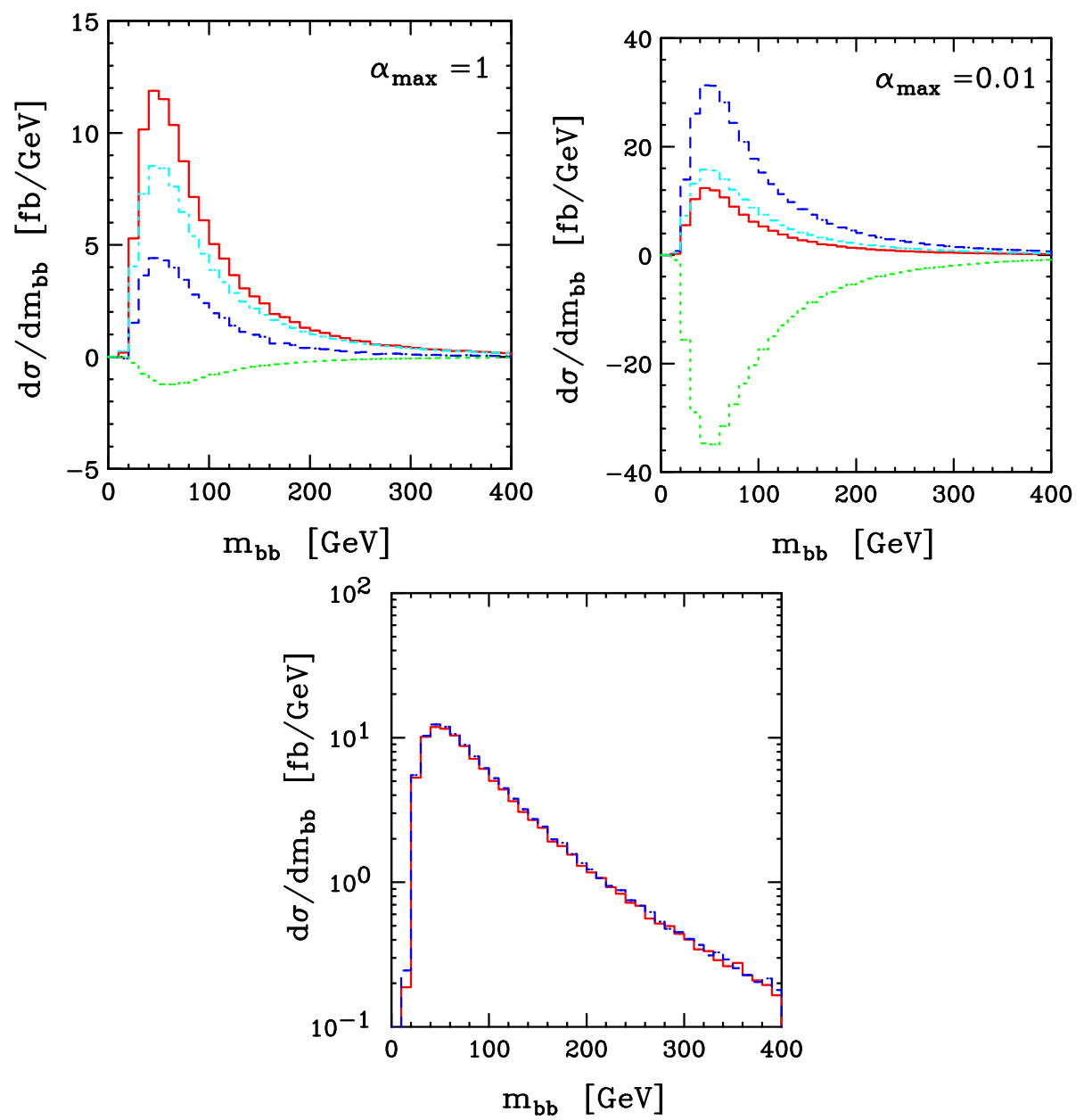

Figure 1: Distribution of the invariant mass $m_{b \bar{b}}$ of the bottom-anti-bottom pair for $p p \rightarrow t \bar{t} b \bar{b}+X$ at the LHC for different parts of the real radiation contribution with different choices of $\alpha_{\max }, \alpha_{\max }=1$ and $\alpha_{\max }=$ 0.01 . The red solid line corresponds to the sum of all contributions, the blue dashed line represents the dipole subtracted real emission, the cyan dot-dashed line corresponds to the sum of the $K$ and $P$ insertion operators, and finally the green dotted line represents the I insertion operator. The sum of all the contributions for the two different choices of $\alpha_{\text {max }}$ is depicted below.

It should be noted that based on this complete calculation, the NLO QCD corrections to arbitrary distributions can be investigated within realistic selection cuts. On the other hand, this calculation served as an important test of the system, as well as a test of the correctness of the preceding results from [5].

\section{NNLO}

The current status of fixed order NNLO calculations for top quark pair production in hadronic collisions can be summarized as follows. The most advanced evaluations concern the virtual corrections. In particular, the latter have been derived in the high energy, fixed angle limit in [7]. Subsequently, the complete result for the quark annihilation channel has been given numerically 

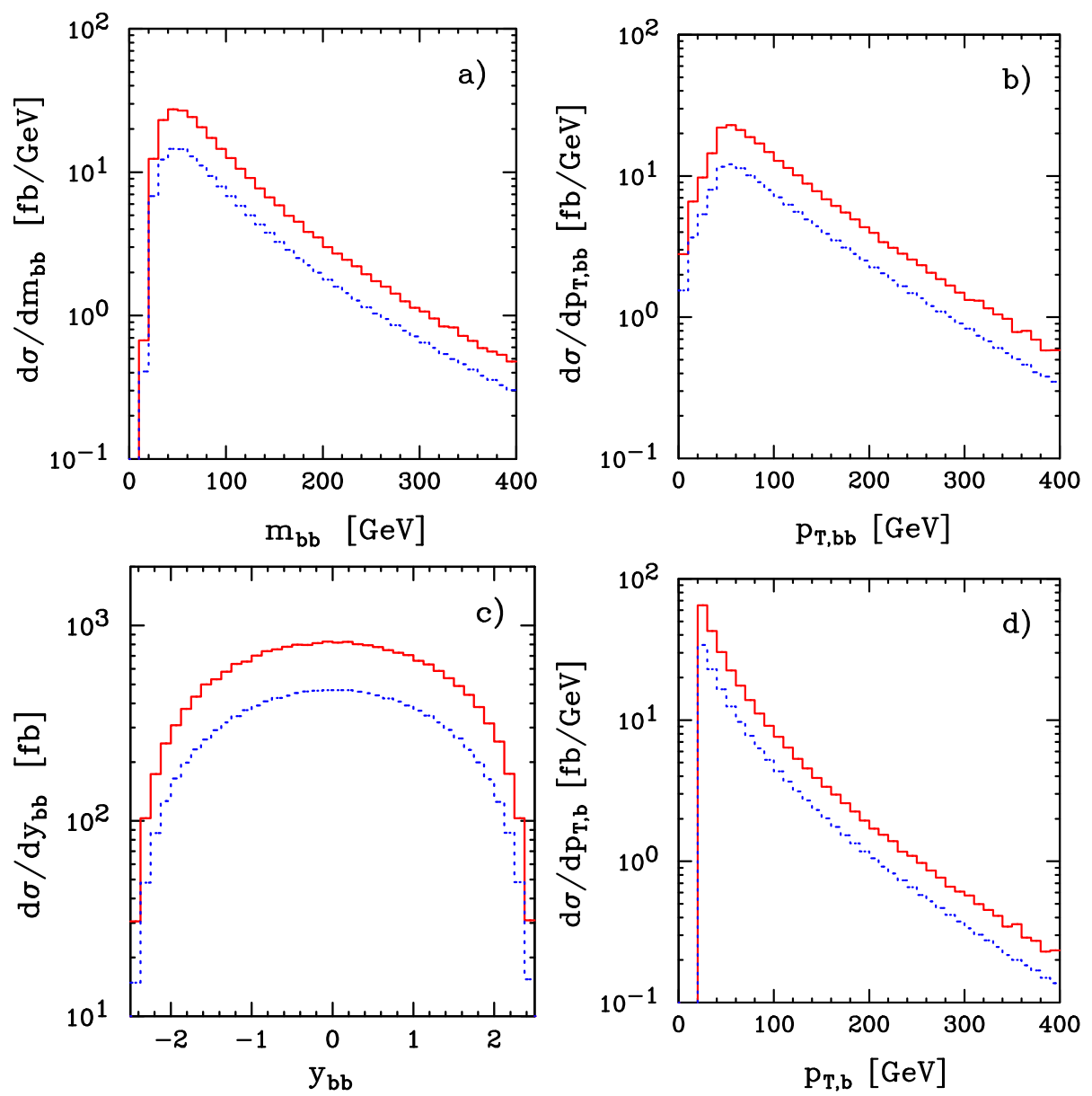

Figure 2: Distribution of the invariant mass $m_{b \bar{b}}$ of the bottom-anti-bottom pair $(a)$, distribution in the transverse momentum $p_{T_{b \bar{b}}}$ of the bottom-anti-bottom pair $(b)$, distribution in the rapidity $y_{b \bar{b}}$ of the bottomanti-bottom pair (c) and distribution in the transverse momentum $p_{T_{b}}$ of the bottom quark (d) for $p p \rightarrow$ $t \bar{t} b \bar{b}+X$ at the LHC at LO (blue dashed line) and NLO (red solid line). All distributions have been obtained with $\alpha_{\text {max }}=0.01$.

in [8], whereas leading color and fermionic contributions in the same channel are known from [9]. Additionally, the complete divergence structure has been given in [10]. Thus, the missing contributions are the finite part of the virtual corrections in the gluon fusion channel, and the real radiation contribution.

While the full calculation is still under way, there has been important progress in the evaluation of the behavior at threshold. A first, albeit incorrect, attempt at a complete NNLO threshold expansion has been presented in [11]. However, it is only in [12] that the known contributions have been put together with some missing ingredients due to potential interactions between the quarks in order to obtain the correct and complete expansion.

Assuming that we decompose the cross section as follows

$$
\sigma_{i j}(\beta, \mu, m)=\sigma_{i j}^{(0)}\left\{1+\frac{\alpha_{s}\left(\mu^{2}\right)}{4 \pi}\left[\sigma_{i j}^{(1)}\right]+\left(\frac{\alpha_{s}\left(\mu^{2}\right)}{4 \pi}\right)^{2}\left[\sigma_{i j}^{(2)}\right]+\mathscr{O}\left(\alpha_{s}^{3}\right)\right\},
$$


where $i, j$ denotes the possible initial states (we concentrate only on gluon fusion, gg, and quark annihilation, qq), and with

$$
\beta=\sqrt{1-4 m_{t}^{2} / s}
$$

we have (we have put the scales $\mu=m$ )

$$
\begin{aligned}
\sigma_{q \bar{q}}^{(2)}= & \frac{3.60774}{\beta^{2}}+\frac{1}{\beta}\left(-140.368 \ln ^{2} \beta+32.106 \ln \beta+3.95105\right) \\
& +910.222 \ln ^{4} \beta-1315.53 \ln ^{3} \beta+592.292 \ln ^{2} \beta+528.557 \ln \beta+C_{q q}^{(2)}, \\
\sigma_{g g}^{(2)}= & \frac{68.5471}{\beta^{2}}+\frac{1}{\beta}\left(496.3 \ln ^{2} \beta+321.137 \ln \beta-8.62261\right) \\
& +4608 \ln ^{4} \beta-1894.91 \ln ^{3} \beta-912.349 \ln ^{2} \beta+2456.74 \ln \beta+C_{g g}^{(2)},
\end{aligned}
$$

Before we discuss the origin of these formulae, let us note that the cross section expansion in the limit, where the emitted gluons are soft (up to two at NNLO), but the final state is not at threshold, has been given in [13].

The results in Eq. (3.3) require the following ingredients: the two-loop soft anomalous dimension at threshold [14, 15], the matching coefficients at the one-loop level in the two different color channels [16] and finally the contribution of the potential interactions between the heavy quarks. The latter can be derived from threshold expansion of the top quark pair production cross section in $e^{+} e^{-}$and $\gamma \gamma$ collisions [17]. For example the contribution for the gluon fusion is the same as that in $\gamma \gamma$ scattering up to some minor modifications. If we take the formula for the $R$ ratio in $\gamma$ collisions, we have

$$
R_{S}^{++}=6 Q_{t}^{4} N_{c} \beta\left(1-\frac{\beta^{2}}{3}\right) \cdot\left[1+C_{F}\left(\frac{\alpha_{s}}{\pi}\right) \Delta^{(1)}+C_{F}\left(\frac{\alpha_{s}}{\pi}\right)^{2} \Delta^{(2)}\right]
$$

where

$$
\begin{aligned}
& \Delta^{(2)}=C_{F} \Delta_{A}+C_{A} \Delta_{N A}+T_{R} N_{L} \Delta_{L}+T_{R} N_{H} \Delta_{H}, \\
& \Delta_{A}=\frac{\pi^{4}}{12 \beta^{2}}+\left(-\frac{5}{2}+\frac{1}{8} \pi^{2}\right) \frac{\pi^{2}}{\beta}+\frac{27}{8} \pi^{2}+\frac{25}{4}+\frac{35}{192} \pi^{4}-2 \pi^{2} \ln (2 \beta)+2 x_{A} ; \\
& \Delta_{N A}=\left(\frac{31}{72}-\frac{11}{12} \ln (2 \beta)\right) \frac{\pi^{2}}{\beta}+\pi^{2}\left(\frac{5}{4}-\ln (2 \beta)\right)+2 x_{N A} ; \\
& \Delta_{L}=\left(-\frac{5}{18}+\frac{1}{3} \ln (2 \beta)\right) \frac{\pi^{2}}{\beta}+2 x_{L} ; \\
& \Delta_{H}=2 x_{H} .
\end{aligned}
$$

From this formula we need to first eliminate the effect of the one-loop matching times the one-loop potential (Coulomb) contribution. This is contained in $\Delta_{A}$ in the term proportional to $1 / \beta$. Next we retain only the terms with enhancements in $\beta$, i.e. $1 / \beta$ or $\log \beta$. Finally, we need to take into account the possible color channels, since in $\gamma$ collisions there is only a singlet contribution, whereas gluon fusion requires also the octet. This is done by the replacement $C_{F} \rightarrow C_{F}-C_{A} / 2$. 

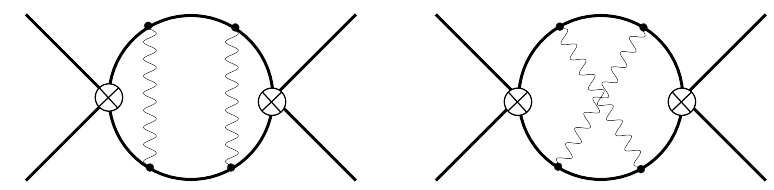

Figure 3: Graphs relevant to the potential contributions in the singlet and octet channels discussed in the text. Crosses correspond to the singlet or octet color-projection operators.

The correctness of the replacement can be checked by considering the color structures depicted in Fig. 3

Ref. [12] contains a more extensive discussion of the problem together with a proof that no other enhancements are present at threshold at this order of the perturbative expansion.

\section{References}

[1] A. Kanaki and C. G. Papadopoulos, Comput. Phys. Commun. 132 (2000) 306; C. G. Papadopoulos, Comput. Phys. Commun. 137 (2001) 247; C. G. Papadopoulos and M. Worek, Eur. Phys. J. C 50 (2007) 843; A. Cafarella, C. G. Papadopoulos and M. Worek, Comput. Phys. Commun. 180 (2009) 1941 ;

[2] A. van Hameren, C. G. Papadopoulos and R. Pittau, JHEP 0909 (2009) 106;

[3] G. Ossola, C. G. Papadopoulos and R. Pittau, Nucl. Phys. B 763 (2007) 147; JHEP 0803 (2008) 042;

[4] M. Czakon, C. G. Papadopoulos and M. Worek, JHEP 0908 (2009) 085;

[5] A. Bredenstein, A. Denner, S. Dittmaier and S. Pozzorini, JHEP 0808 (2008) 108; Phys. Rev. Lett. 103 (2009) 012002;

[6] G. Bevilacqua, M. Czakon, C. G. Papadopoulos, R. Pittau and M. Worek, JHEP 0909 (2009) 109;

[7] M. Czakon, A. Mitov and S. Moch, Phys. Lett. B 651 (2007) 147; Nucl. Phys. B 798 (2008) 210;

[8] M. Czakon, Phys. Lett. B 664 (2008) 307;

[9] R. Bonciani, A. Ferroglia, T. Gehrmann, D. Maitre and C. Studerus, JHEP 0807 (2008) 129;

R. Bonciani, A. Ferroglia, T. Gehrmann and C. Studerus, JHEP 0908 (2009) 067;

[10] A. Ferroglia, M. Neubert, B. D. Pecjak and L. L. Yang, Phys. Rev. Lett. 103 (2009) 201601; JHEP 0911 (2009) 062;

[11] S. Moch and P. Uwer, Phys. Rev. D 78 (2008) 034003;

[12] M. Beneke, M. Czakon, P. Falgari, A. Mitov and C. Schwinn, arXiv:0911.5166 [hep-ph].

[13] V. Ahrens, A. Ferroglia, M. Neubert, B. D. Pecjak and L. L. Yang, arXiv:0912.3375 [hep-ph].

[14] M. Beneke, P. Falgari and C. Schwinn, Nucl. Phys. B 828 (2010) 69;

[15] M. Czakon, A. Mitov and G. Sterman, Phys. Rev. D 80 (2009) 074017;

[16] K. Hagiwara, Y. Sumino and H. Yokoya, Phys. Lett. B 666 (2008) 71; M. Czakon and A. Mitov, Nucl. Phys. B 824 (2010) 111; Phys. Lett. B 680 (2009) 154;

[17] A. Czarnecki and K. Melnikov, Phys. Rev. Lett. 80 (1998) 2531; Phys. Rev. D 65 (2002) 051501 